Muro de la Investigación, 2018: 3(1), Enero-Julio, ISSN: 2523-2886

DOI:https://doi.org/10.17162/rmi.v3i1.1112

\title{
Inteligencia emocional y rendimiento académico en estudiantes de nivel primario en una institución educativa confesional de la selva peruana
}

\author{
Dámaris Pinedo Chambi $^{1 \mathrm{a}}$ y Sara Esther Richard Pérez ${ }^{2}$
}

Universidad Peruana Unión, Perú ${ }^{12}$

\author{
Recibido: 03 de febrero de $2018 \quad$ Aceptado: 05 de julio de 2018
}

\section{Resumen}

El objetivo del estudio fue establecer la relación entre inteligencia emocional y rendimiento académico en estudiantes del nivel primario de una institución educativa confesional de la selva peruana. Se trata de un estudio tipo descriptivo correlacional, no experimental de corte trasversal. Para medir la inteligencia emocional se aplicó el Inventario de Inteligencia Emocional de Baron ICE NA, adaptado en Perú por Ugarriza y Pajares. (2003) y para rendimiento académico se tomaron en cuenta las notas de las áreas de Comunicación y Matemática. La población estuvo conformada por 235 estudiantes de tercero a sexto grado de primaria, cuyas edades oscilan entre 8 a 13 años, de ambos sexos. Se encontró que no existe relación significativa entre la inteligencia emocional y el rendimiento académico en el área de comunicación, pero sí hay relación significativa en el área de matemática. En cuanto a las dimensiones se halló que no existe relación significativa entre la inteligencia intrapersonal, adaptabilidad, manejo de estrés y el rendimiento académico en las áreas de comunicación y matemática; sin embargo, sí existe relación de la inteligencia interpersonal y el rendimiento académico en el área de matemática.

Palabras clave: inteligencia emocional, intrapersonal, interpersonal, manejo de estrés, rendimiento académico, comunicación y matemática.

\section{Emotional intelligence and academic performance in elementary school students in a confessional educational institution in the Peruvian jungle}

\footnotetext{
Abstract

The objective of the study was to establish the relationship between emotional intelligence and academic performance in primary school students in a confessional educational institution in the 
Peruvian jungle. It is a correlational descriptive, non-experimental cross-sectional type study. To measure emotional intelligence, the Emotional Intelligence Inventory from Baron ICE NA was used, as adapted in Peru by Ugarriza and Pajares (2003), and for academic performance the final grades for the courses of Communication and Mathematics were used. The population consisted of 235 students from third to sixth grade of primary school, whose ages range from 8 to 13 years, of both sexes. It was found that there is no significant relationship between emotional intelligence and academic performance in the area of communication, but there is a significant relationship in the area of mathematics. Regarding the dimensions, it was found that there is no significant relationship between intrapersonal intelligence, adaptability, stress management and academic performance in the areas of communication and mathematics; However, there is a relationship between interpersonal intelligence and academic performance in the area of mathematics.

Key-words: emotional intelligence, intrapersonal, interpersonal, stress management, academic performance, communication and mathematics.

\section{Introducción}

En la antigüedad, la cultura occidental tenía como dogma fundamental la inteligencia, esto fue aproximadamente hasta los principios del siglo XX donde se formularon las primeras teorías psicológicas de la inteligencia (Pueyo, 1997). Sin embargo, a mediados del siglo XX se produjo un descrédito a los test de inteligencia, ya que solo medían las capacidades lingüísticas, matemáticas, ciencia, etc. (Martín, 2003)

Debido a estas dificultades surge un concepto diferente respecto a la inteligencia, que recibe el nombre de inteligencia emocional, la cual comprende aptitudes positivas como personalidad, habilidades sociales, empatía, control de impulsos y solución de problemas.

A esto añade Shapiro (1997), la necesidad de que los padres se involucren y refuercen las capacidades emocionales de sus hijos, ya que no siempre los niños desarrollan de forma espontánea las cualidades emocionales y sociales. Muchos descubrimientos sugieren que la vinculación temprana con la familia, en especial con la madre, pueden tener impactos sustanciales sobre las emociones y la personalidad subsecuentes en los niños (Rodríguez, 2004). Sin embargo, Vallés y Vallés (2003) consideran que la escuela también forma parte del desarrollo emocional del menor ya que pasa en las aulas gran parte de su infancia y adolescencia, el profesor/tutor llega a ser uno de sus referentes más importantes en cuanto a actitudes, comportamientos, emociones y sentimientos puesto que no solo brinda conocimientos teóricos, sino que también estimula la creación de ambientes (dinámicas, trabajos en grupo, etc.) que propician el desarrollo de las capacidades socio-emocionales, búsqueda de soluciones ante los conflictos interpersonales y 
enseñanza de habilidades empáticas como prestar atención en clases, saber escuchar y comprender los puntos de vista de los demás.

Desde la práctica profesional docente se dice que una variable que se relaciona significativamente con la inteligencia emocional es el rendimiento académico, Jiménez (2000) define el rendimiento académico como nivel de conocimiento respecto a un área o materia, la cual es superior a su edad y nivel académico. Por su parte, Novaez (1986) señala que el rendimiento académico guarda relación con la aptitud, y este sería el resultado con otros factores intervinientes como los volitivos, afectivos y emocionales.

López (2008), clasifica en dos grupos los factores que intervienen en el rendimiento académico: factores exógenos, que son aquellas condiciones externas en las que se desarrolla y desenvuelve el estudiante, como el ambiente familiar, los profesores, nivel socioeconómico, espacio físico, entre otros. Los factores endógenos se refieren a las características inherentes del individuo como la personalidad, motivación e inteligencia racional y emocional (Benites, Gimenez y Osicka, 2000). Por su parte, Goleman (1995) afirma que tenemos dos cerebros, dos mentes y dos clases diferentes de inteligencias, la racional y la emocional. Nuestro desempeño en la vida está determinado por ambas, no solo es importante el coeficiente intelectual (CI), sino también la inteligencia emocional (IE).

En relación a esto, estudios realizados en distintas partes de Sudamérica y el Perú se comprobó que existe relación entre inteligencia emocional y rendimiento académico, por ejemplo, Arévalo y Escalante (2004) realizaron una investigación para determinar la relación que existe entre la inteligencia emocional y el rendimiento académico en estudiantes de cuarto y quinto grado de secundaria, utilizando como instrumento el inventario de cociente emocional Baron (I-CE). Los resultados demostraron que existe relación significativa entre el cociente emocional y el rendimiento académico.

No obstante, existen otras investigaciones que demuestran que no hay relación o existe una débil relación entre las variables, por ejemplo: Castellano, (2010) en una investigación realizada sobre la relación entre inteligencia emocional y comprensión lectora en estudiantes de quinto y sexto grado de primaria del distrito del Callao, demostró que existe relación débil entre la Inteligencia Emocional y la comprensión lectora. No existe relación entre la inteligencia intrapersonal e interpersonal con la comprensión lectora. Sin embargo, si existe relación significativa entre la adaptabilidad, manejo del estrés con la comprensión lectora. 
Este estudio observó niños que fácilmente lloran, niños que tienen reacciones inadecuadas que generan conflictos con los demás, físico como verbal y que no saben reconocer sus errores y tampoco pedir disculpas. Algunos niños de este estudio mostraron conductas cohibidas, baja autoestima y hogares disfuncionales, donde padres ausentes física y emocionalmente no se dan tiempo para ayudar a los niños en sus actividades escolares y menos aún brindar el soporte emocional que esta edad requieren. Esto en algunas ocasiones afecta su rendimiento académico, mostrando desánimo al cumplir con sus deberes escolares, poca motivación e interés en los resultados académicos y por ende pueden presentar bajas calificaciones (Gilly, 1978).

Esta investigación tiene como objetivo principal analizar la relación que existe entre la inteligencia emocional y el rendimiento académico en los estudiantes de tercero a sexto grado de primaria de una institución educativa confesional de la selva peruana.

\section{Metodología}

El estudio es de tipo descriptivo correlacional. Descriptivo, porque se medirán las variables en estudio. Correlacional, porque se realizarán pruebas estadísticas de correlación con el propósito de determinar la significatividad de las mismas (Hernández, Fernández y Baptista, 2006).

Participantes

Para la determinación de la muestra se realizó un muestreo no probabilístico, por conveniencia considerando criterios de inclusión y exclusión. Tomando en cuenta los criterios de exclusión, la muestra quedó conformada por un total de 203 estudiantes de tercero a sexto grado de primaria, cuyas edades oscilan entre 8 a 13 años, de ambos sexos.

Instrumentos

Se utilizaron dos instrumentos para la evaluación de la inteligencia emocional y rendimiento académico. A continuación, se detalla la autoría de los mismos y los resultados de las correspondientes pruebas de validez y consistencia.

Inventario emocional de Baron ICE: NA-Completo

El inventario comprende cinco componentes: intrapersonal, interpersonal, adaptabilidad, manejo del estrés y estado de ánimo general. Para la interpretación de los puntajes del Baron ICE: NA se examinaron los patrones de los puntajes estándares: 
- $\quad 130$ y más: Capacidad emocional y social atípica. Excelentemente desarrollada.

- $\quad 120$ a 129: Capacidad emocional y social muy alta. Muy bien desarrollada.

- $\quad 110$ a 119: Capacidad emocional y social alta. Bien desarrollado.

- $\quad 90$ a 109: Capacidad emocional y social adecuada. Promedio.

- $\quad 80$ a 89: Capacidad emocional y social baja. Mal desarrollado.

- 70 a 79: Capacidad emocional y social muy baja. Necesita mejorarse considerablemente.

- $\quad 69$ y menos: Capacidad emocional y social atípica y deficiente. Nivel de desarrollo marcadamente bajo.

La fiabilidad global de la escala y de sus dimensiones se valoró calculando el índice de la consistencia interna mediante el coeficiente Alpha de Cronbach. La tabla 11 permite apreciar que la consistencia interna global de la escala (60 ítems) en la muestra estudiada es de .900 que puede ser valorado como indicador de una elevada fiabilidad. Los coeficientes de correlación ProductoMomento de Pearson (r) son significativos, lo cual confirma que la escala presenta validez de constructo. Los coeficientes que resultan de la correlación entre cada una de las dimensiones y el constructo en su globalidad son moderados en su mayoría, además de ser altamente significativos.

Registro de notas de los estudiantes de tercero a sexto de primaria

El rendimiento académico de los estudiantes se obtendrá a partir de los resultados que brindará la institución educativa, a través de las Actas Oficiales de Evaluación de cada grado y sección en el área de Comunicación y Matemática, las calificaciones son cualitativas del siguiente modo:

- $\quad$ Logro destacado (AD)

- $\quad$ Logro previsto (A)

- $\quad$ En proceso (B)

- $\quad$ En inicio (C)

Análisis de datos 
Para el análisis de los datos obtenidos se utilizó el paquete estadístico para las ciencias sociales o SPSS por sus siglas en inglés (Statistical Package for the Social Sciences), versión 22, alli se realizaron las tablas descriptivas, usando las variables de estudio, se aplicó la técnica del chi - cuadrado para estudiar la relación entre las variables: inteligencia emocional y rendimiento académico en las áreas de comunicación y matemática.

\section{Resultados}

En los resultados se resume los datos compilados y el análisis de los datos que sean relevantes el discurso, presente con detalle los datos a fin de que pueda justificar las conclusiones.

\section{Relación entre inteligencia emocional y rendimiento académico en comunicación}

Se aprecia en la tabla 01 que no existe relación significativa entre el nivel de inteligencia emocional y el rendimiento académico en comunicación $(\mathrm{x} 2=11.948, \mathrm{p}>.05)$, es decir el grado de inteligencia emocional de los estudiantes no guarda relación con el nivel de rendimiento alcanzado en esta área ( $\mathrm{AD}, \mathrm{A}, \mathrm{B}$ y $\mathrm{C})$. De manera similar tampoco está asociado con todos los componentes de la inteligencia emocional.

Tabla 1

Relación entre inteligencia emocional y rendimiento académico en comunicación

\begin{tabular}{lccc}
\hline & $\mathrm{x}^{2}$ & $\mathrm{gl}$ & $\mathrm{P}$ \\
\hline Inteligencia emocional & 11.948 & 6 & .063 \\
Intrapersonal & 5.818 & 6 & .444 \\
Interpersonal & 12.484 & 6 & .052 \\
Adaptabilidad & 7.725 & 6 & .259 \\
Manejo de estrés & 10.305 & 6 & .112 \\
\hline
\end{tabular}

Fuente: propia

\section{Relación entre inteligencia emocional y rendimiento académico en matemática}

Se observa en la tabla 2 que existe relación significativa entre el grado de inteligencia emocional y el nivel de rendimiento académico en matemática $(\mathrm{x} 2=17.239, \mathrm{p}<.05)$. Asimismo, existe relación significativa entre el nivel de inteligencia intrapersonal y el nivel de rendimiento académico en matemática $(\mathrm{x} 2=13.340, \mathrm{p}<.05)$. No obstante, se aprecia que no existe relación 
significativa entre los demás componentes de la inteligencia emocional y el desempeño en matemática.

\section{Tabla 2}

Relación entre inteligencia emocional y rendimiento académico en matemática

\begin{tabular}{lccc}
\hline & $\mathrm{x} 2$ & $\mathrm{~g} 1$ & $\mathrm{p}$ \\
\hline Inteligencia emocional & 17.239 & 6 & .008 \\
Intrapersonal & 5.194 & 6 & .519 \\
Interpersonal & 13.340 & 6 & .038 \\
Adaptabilidad & 10.092 & 6 & .121 \\
Manejo de estrés & 9.217 & 6 & .162 \\
\hline
\end{tabular}

Fuente: propia

\section{Conclusión}

De los resultados de la presente investigación se extraen las siguientes conclusiones:

El objetivo general tuvo como finalidad analizar si existe relación significativa entre la inteligencia emocional y el rendimiento académico, y para el análisis de los resultados se tomó en cuenta los resultados obtenidos de las áreas de comunicación y matemática, ya que a estos se consideran los pilares del aprendizaje.

En relación al análisis de correlación de la inteligencia emocional y el rendimiento académico en el área de comunicación se halló que no existe relación significativa entre ambas variables (x2 $=11.948, \mathrm{p}>.063)$.

Por otro lado, se encontró que sí existe relación significativa entre la variable de inteligencia emocional y rendimiento académico en el área de matemática $(x 2=17.239, \mathrm{p}<.008)$.

Referente a las dimensiones de la inteligencia emocional se pudo evidenciar que no existe una relación significativa entre la dimensi académico en el área de comunicación 5.194, $\mathrm{p}>.519)$.

Referente a la dimensión de la intelį̧ de comunicación no existe relacion (x2 evidenciar que sí existe relación signific

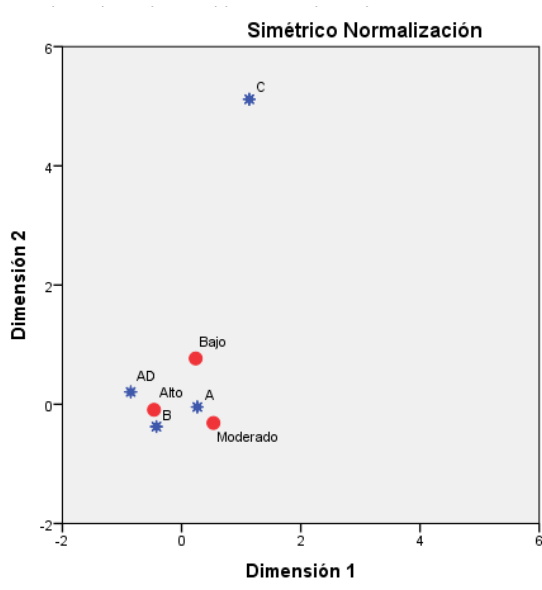

1 rendimiento Interpersonal
* Matemática emática $(\mathrm{x} 2=$ nico en el área nática se pudo 
Por otro lado respecto a la dimensión de adaptabilidad los resultados demostraron que no existe relación significativa con el rendimiento académico en el área de comunicación $(x 2=7.725, \mathrm{p}>$ .259), ni en el área de matemática $(x 2=10.092, \mathrm{p}>.121)$.

Finalmente en relación a la dimensión manejo de estrés, los resultados demostraron que no existe relación significativa con el rendimiento académico en el área de comunicación $(\mathrm{x} 2=$ $10.305, \mathrm{p}>.112)$, ni en el área de matemática $(\mathrm{x} 2=9.217, \mathrm{p}>.162)$.

\section{Recomendaciones}

De esta investigación se extraen las siguientes recomendaciones:

Ampliar la presente investigación a fin de encontrar otras variables como el clima social familiar que influyen en el rendimiento académico, puesto que la familia ejerce una fuerte influencia en la configuración del comportamiento escolar del niño.

Desarrollar esta investigación en una población distinta como por ejemplo en instituciones educativas públicas. Esto con el fin de identificar si hay diferencias significativas para atender a los estudiantes de acuerdo a sus oportunidades y necesidades.

Considerar realizar la asociación de inteligencia emocional y rendimiento académico en los años posteriores, es decir los estudiantes de secundaria porque sería interesante saber si las habilidades relacionadas a la inteligencia emocional y el rendimiento académico tienen diferencias a lo largo del tiempo y conforme el estudiante va madurando.

Extender la población de participantes con el objetivo de conseguir una mayor confiabilidad de los instrumentos aplicados y cuyos resultados sirvan como base para tomar medidas correctivas en beneficio de los estudiantes de esta zona del país.

Identificar en investigaciones posteriores si existe otras variables que puedan estar relacionadas con el rendimiento académico y la inteligencia emocional independientemente, porque como se observa en este estudio los resultados demostraron que no existe relación entre ambas, sin embargo hay estudios que exponen que si hay, esto puede deberse como lo afirma la teoría a otros factores que intervienen.

Considerar la religión a fin de saber si esta es una variable que puede influir en el desarrollo de la inteligencia emocional y el rendimiento académico de los estudiantes. 
Continuar fomentando en la institución educativa el desarrollo de las habilidades emocionales y cognitivas a través de la acción tutorial y también con capacitaciones a los padres y maestros que son los que están en una relación más directa con el estudiante.

\section{Referencias}

Arévalo, M. y Escalante, M. (2004). Relación entre inteligencia emocional y rendimiento académico en alumnos de cuarto y quinto grado de E.B.R en colegios estatales del distrito de Barranco. (Tesis de Licenciatura). Universidad Femenina del Sagrado Corazón. Perú.

Benítez, M., Gimenez, M. y Osicka, R. (2000). Las asignaturas pendientes y el rendimiento académico: ¿existe alguna relación?. Revista Electrónica Iberoamericana sobre Calidad, Eficacia y Cambio en Educación 2(1), 254-270.

Castellano, J. (2010). Inteligencia Emocional y Comprensión Lectora en alumnos del $6^{\circ}$ Grado de Primaria de la RED N ${ }^{\circ}$ Distrito del Callao. (Tesis de Maestría). Universidad San Ignacio de Loyola. Callao, Perú.

Gilly, M. (1978). El problema del rendimiento escolar. Barcelona: Oikos-Tau

Goleman, D. (1995). La inteligencia Emocional. Argentina: Verlap S.A.

Hernández, R., Fernández, C. y Baptista, P. (2006). Metodología de la investigación, cuarta edición. México: McGraw Hill/ Interamerican Editores.

Jiménez, M. (2000) Competencia social: intervención preventiva en la escuela. Universidad de Alicante. Infancia y sociedad. 24, 21-48. Recuperado de http://www.scielo.br/scielo.php?script=sci_nlinks\&ref=000317\&pid=S01044036201400030000400040\&lng=en

López, M (2008). La Inteligencia emocional y las estrategias de aprendizaje como predictores del rendimiento académico en estudiantes universitarios. (Tesis de maestría en psicología educativa). Universidad Nacional Mayor de San Marcos, Lima- Perú.

Martín, M. (2007). Análisis histórico y conceptual de las relaciones entre la inteligencia y la razón. (Tesis Doctoral). Universidad De Málaga, España. Recuperado de: http://www.biblioteca.uma.es/bbldoc/tesisuma/1685391x.pdf

Novaez, M (1986). Psicología de la actividad escolar. México: Editorial Iberoamericana.

Pueyo, A. (1997) Manual de Psicología Diferencial. Madrid: McGraw-Hill.

Rodríguez, J., y Gallego S. (1992). Lenguaje y rendimiento académico en educación secundaria. (Tesis de Licenciatura). Universidad de Salamanca. España.

Shapiro, L. (1997). La inteligencia emocional de los niños. España: Grafo, S.A.

Ugarriza, N. y Pajares, L. (2005) La evaluación de la inteligencia emocional a través del inventario de BarOn ICE: NA, en una muestra de niños y adolescentes. Redalyc, Núm. 8, pp. 11-58. Recuperado de: http://www.redalyc.org/html/1471/147112816001/ 
Vallés, A. y Vallés, C. (2003). Psicopedagogía de la Inteligencia Emocional. Valencia: Promolibro. 